\title{
Oscillating system design applying universal formula for control
}

\author{
Efimov D., Perruquetti W.
}

\begin{abstract}
The problem of oscillating system design applying the homogeneity approach is studied. The Anti-control Lyapunov Function (ALF) is introduced as a counterpart of Control Lyapunov Function (CLF) for the control design that destabilizes a nonlinear system. A universal anti-control formula is proposed. Next, the universal control formulas based on ALF and CLF are used to design an oscillating system. Efficiency of the proposed approach is demonstrated on example.
\end{abstract}

\section{INTRODUCTION}

The problem of a stabilizing control design for nonlinear dynamical systems was intensively studied during the last few decades [15]. A number of approaches have been developed: method of CLF [2], [26], feedback linearization approach [14], passification design method, flatness-based control [10], [11], backstepping, nested saturation design or forwarding [16], [22]. Almost all these techniques have been devoted to nonlinear systems stabilization with respect to an equilibrium or a reference trajectory. Another promising topic deals with the problem of nonlinear systems stabilization with respect to a set [12], [21], [23], [24], [25], [28] (part of variables or output). Such problem arises in oscillations or synchronization control, energy level stabilization in mechanical systems, maneuvering problem or in robotic applications.

In spite of these achievements the design problem of a system with a predefined number of limit cycles, their locations and frequencies of oscillation remains rather challenging and unsolved. This problem can be considered as an inverse of the Hilbert's 16th problem that addresses the issue of evaluation of an upper bound for the number of limit cycles in polynomial vector fields of degree $n$ and investigation of their relative positions (for a planar system).

Partly such a lack of solutions can be linked with the absence of an apparatus for complex nonlinear oscillating system analysis and, hence, design. Almost all conventional design techniques for nonlinear systems are based on the Lyapunov second method, that is hard to apply for oscillating or chaotic system analysis. Recently revisited the Yakubovich's oscillation framework gives some promising hints and methods for complex oscillatory system analysis

The first author is with University of Bordeaux, IMS-lab, Automatic control group 351 cours de la libération, 33405 Talence, France, denis.efimov@ims-bordeaux.fr. The second author is with LAGIS (UMRCNRS 8146), Ecole Centrale de Lille, BP 48, Cité Scientifique, 59651 Villeneuve-d'Ascq, France and ALIEN project at INRIA - LNE, Parc Scientifique de la Haute Borne 40, avenue Halley Bât.A, Park Plaza 59650 Villeneuve d'Ascq, France, wilfrid.perruquetti@inria.fr. and design [29], [7]. This concept of oscillations covers all types of oscillating behavior: from periodical to chaotic. Combination of this approach with the homogeneous system theory simplifies its applicability conditions [8]. In this work we are going to develop these frameworks and apply the homogeneity approach for synthesis of oscillating and chaotic systems.

All notations are summarized in Section 2. The preliminary definitions are given in Section 3. The control design is investigated in Section 4. Example of application of the proposed technique follows the theoretical development in Section 5.

\section{NotATIONS}

- $\mathbb{R}_{+}=\{x \in \mathbb{R}: x>0\}$, where $\mathbb{R}$ is the set of real number.

- the sequence $i=1, \ldots, n$ is denoted as $i=\overline{1, n}$.

- a continuous function $\alpha: \mathbb{R}_{+} \rightarrow \mathbb{R}_{+}$belongs to the class $\mathcal{K}$ if $\alpha(0)=0$ and the function is strictly increasing. The function $\alpha: \mathbb{R}_{+} \rightarrow \mathbb{R}_{+}$belongs to the class $\mathcal{K}_{\infty}$ if $\alpha \in \mathcal{K}$ and it is increasing to infinity.

- $|s|$ is stated for the absolute value of $s \in \mathbb{R},\|\cdot\|$ denotes a norm on $\mathbb{R}^{n}$ (the Euclidean norm by default if another is not stated explicitly).

- the notation $D V(x) f(x)$ stands for the directional derivative of a continuously differentiable function $V$ with respect to a vector field $f$ evaluated at the point $x$, and for Dini derivative in the direction of $f$ for a locally Lipschitz function $V$ :

$$
D V(x) f(x)=\lim \inf _{t \rightarrow 0+} \frac{V(x+t f(x))-V(x)}{t} .
$$

\section{Preliminaries}

Consider the following nonlinear system:

$$
\dot{x}=f(x),
$$

where $x \in \mathbb{R}^{n}$ is the state vector, $f: \mathbb{R}^{n} \rightarrow \mathbb{R}^{n}$ is a locally Lipschitz continuous function. For an initial condition $x_{0} \in$ $\mathbb{R}^{n}$ the corresponding solution $x\left(t, x_{0}\right)$ of the system (1) is defined at least locally in time $t \geq 0$.

\section{A. Homogeneity}

In the following, for any positive real number $\lambda$ and any strictly positive numbers $r_{i}, i \in \overline{1, n}$ called weights one can define: 
- the vector of weights $r=\left(r_{1}, \ldots, r_{n}\right)^{T}$,

- the dilation matrix

$$
\Lambda_{r}=\operatorname{diag}\left\{\lambda^{r_{i}}\right\}_{i=1}^{n},
$$

note that for $x \in \mathbb{R}^{n}$ we have $\Lambda_{r} x=$ $\left(\lambda^{r_{1}} x_{1}, \ldots, \lambda^{r_{i}} x_{i}, \ldots, \lambda^{r_{n}} x_{n}\right)^{T}$.

- let $\mathbf{r}=\prod_{i=1}^{n} r_{i}$, then the r-homogeneous norm of $x \in$ $\mathbb{R}^{n}$ is defined by:

$$
\|x\|_{r}=\left(\sum_{i=1}^{n}\left|x_{i}\right|^{\frac{\mathbf{r}}{r_{i}}}\right)^{\frac{1}{\mathbf{r}}} .
$$

Definition 1. A function $h: \mathbb{R}^{n} \rightarrow \mathbb{R}$ is r-homogeneous with degree $d_{r, h} \in \mathbb{R}$ if for all $x \in \mathbb{R}^{n}$ we have (see [13]):

$$
\lambda^{-d_{r, h}} h\left(\Lambda_{r} x\right)=h(x) .
$$

When such a property holds, we write $\operatorname{deg}_{r}(h)=d_{r, h}$.

Let us note that for any positive real number $\lambda$ :

$$
\left\|\Lambda_{r} x\right\|_{r}=\lambda\|x\|_{r},
$$

thus $\operatorname{deg}_{r}\left(\|\cdot\|_{r}\right)=1$. Let us introduce the following compact set

$$
S_{r}=\left\{x \in \mathbb{R}^{n}:\|x\|_{r}=1\right\} .
$$

Lemma 2. For all $x \in \mathbb{R}^{n}$, the following holds

$$
\begin{gathered}
\min _{\|x\|=1}\|x\|_{r} \times\|x\| \leq\|x\|_{r} \leq \max _{\|x\|=1}\|x\|_{r} \times\|x\| \\
m_{1}\|x\|_{r} \leq\|x\| \leq m_{2}\|x\|_{r},
\end{gathered}
$$

where $m_{1}=\frac{1}{\max _{\|x\|=1}\|x\|_{r}}, m_{2}=\frac{1}{\min _{\|x\|=1}\|x\|_{r}}$,

$$
x \in S_{r} \Longrightarrow m_{r} \leq\|x\| \leq M_{r} .
$$

where $m_{r}=\frac{\min _{x \in S_{r}}\|x\|_{r}}{\max _{\|x\|=1}\|x\|_{r}}, M_{r}=\frac{\max _{x \in S_{r}}\|x\|_{r}}{\min _{\|x\|=1}\|x\|_{r}}$.

Proof: Since $\|x\|_{r}$ is $r$-homogeneous of degree 1 we have $\|x\|_{r}=\|x\|\left\|\frac{x}{\|x\|}\right\|_{r}$, using continuity of $\|x\|_{r}$ and compactness of the unit ball one get the first relation. The two other ones comes directly from the first relation.

The homogeneity notion can be also defined for vector fields or ordinary differential systems as follows.

Definition 3. A vector field $f: \mathbb{R}^{n} \rightarrow \mathbb{R}^{n}$ is $r$-homogeneous with degree $d_{r, f} \in \mathbb{R}$, with $d_{r, f}>-\min _{i \in\{1, \ldots, n\}} r_{i}$ if for all $x \in \mathbb{R}^{n}$ we have (see [13]):

$$
\lambda^{-d_{r, f}} \Lambda_{r}^{-1} f\left(\Lambda_{r} x\right)=f(x),
$$

which is equivalent for $i$-th component of $f_{i}$ being $r-$ homogeneous function of degree $r_{i}+d_{r, f}$. When such a property holds, we write $\operatorname{deg}_{r}(f)=d_{r, f}$. The system (1) is $r$-homogeneous of degree $d_{r, f}$ if the vector field $f$ is homogeneous of degree $d_{r, f}$.

Theorem 4. [20] For the system (1) with r-homogeneous and continuous function $f$ the following properties are equivalent:

- the system (1) is asymptotically stable;
- there exists a continuously differentiable $r-$ homogeneous Lyapunov function $V: \mathbb{R}^{n} \rightarrow \mathbb{R}_{+}$ such that for all $x \in \mathbb{R}^{n}$,

$$
\begin{array}{cc}
\alpha_{1}(\|x\|) \leq & V(x) \quad \leq \alpha_{2}(\|x\|), \\
D V(x) f(x) & \leq-\alpha(\|x\|), \\
\lambda^{-d} V\left(\Lambda_{r} x\right) & =V(x), d \geq 0,
\end{array}
$$

for some $\alpha_{1}, \alpha_{2} \in \mathcal{K}_{\infty}$ and $\alpha \in \mathcal{K}$.

\section{B. Local homogeneity}

The $r$-homogeneity concept presented in definitions 1,3 and Theorem 4 is introduced for some $r$ and all $\lambda>0$. Restricting the set of admissible values for $\lambda$ we can introduce local homogeneity [8] (this property is in fact has been already defined in the homogeneity in the bi-limit [1], which consist in local homogeneity at the origin and at infinity).

Definition 5. A function $h: \mathbb{R}^{n} \rightarrow \mathbb{R}$ with $h(0)=0$ is $\left(r_{0}, \lambda_{0}, h_{0}\right)$-homogeneous with degree $d_{r_{0}, h_{0}} \in \mathbb{R}$ with $h_{0}(0)=0$ if for all $x \in S_{r_{0}}$ we have:

$$
\lim _{\lambda \rightarrow \lambda_{0}}\left(\lambda^{-d_{r_{0}, h_{0}}} h\left(\Lambda_{r_{0}} x\right)-h_{0}(x)\right)=0 .
$$

A vector field $f: \mathbb{R}^{n} \rightarrow \mathbb{R}^{n}$ with $f(0)=0$ is $\left(r_{0}, \lambda_{0}, f_{0}\right)$ homogeneous with degree $d_{r_{0}, f_{0}}>-\min _{i \in\{1, \ldots, n\}}\left(r_{0 i}\right)$ and $f_{0}(0)=0$ if for all $x \in S_{r_{0}}$ we have:

$$
\lim _{\lambda \rightarrow \lambda_{0}}\left(\lambda^{-d_{r_{0}, f_{0}}} \Lambda_{r_{0}}^{-1} f\left(\Lambda_{r_{0}} x\right)-f_{0}(x)\right)=0,
$$

The system (1) is $\left(r_{0}, \lambda_{0}, f_{0}\right)$-homogeneous with degree $d_{r_{0}, f_{0}} \in \mathbb{R}$ if the vector field $f$ is $\left(r_{0}, \lambda_{0}, f_{0}\right)$-homogeneous with degree $d_{r_{0}, f_{0}} \in \mathbb{R}$.

The coefficients $r_{0 i}>0, i \in \overline{1, n}$ are called the weights, $d_{r_{0}, h_{0}}$ (respectively $d_{r_{0}, f_{0}}$ ) is the degree of homogeneity (it may depend on $\lambda_{0}$ ) and $h_{0}$ (respectively $f_{0}$ ) is the approximating function of $h$ (respectively $f$ ) at $\lambda_{0}$.

If the pairs of functions $\left(h, h_{0}\right)$, and $\left(f, f_{0}\right)$ are continuous, then for any $\varepsilon>0$ there exist $\underline{\lambda}_{\varepsilon} \leq \lambda_{0} \leq \bar{\lambda}_{\varepsilon}$ such that for all $\lambda \in\left[\underline{\lambda}_{\varepsilon}, \bar{\lambda}_{\varepsilon}\right]$ :

$$
\begin{aligned}
\sup _{x \in S_{r_{0}}}\left\|\lambda^{-d_{r_{0}, h_{0}}} h\left(\Lambda_{r_{0}} x\right)-h_{0}(x)\right\| & \leq \varepsilon, \\
\sup _{x \in S_{r_{0}}}\left\|\lambda^{-d_{r_{0}, f_{0}}} \Lambda_{r_{0}}^{-1} f\left(\Lambda_{r_{0}} x\right)-f_{0}(x)\right\| & \leq \varepsilon .
\end{aligned}
$$

In the paper [1] this definition has been introduced for $\lambda_{0}=0$ and $\lambda_{0}=\infty$ (the function $h$ is called homogeneous in the bi-limit if it is simultaneously $\left(r_{0}, 0, h_{0}\right)$-homogeneous and $\left(r_{\infty},+\infty, h_{\infty}\right)$-homogeneous). The system (1) can be also homogeneous in more than two limits

Definition 6. The function $g$ (respectively the vector field $f$, the system (1)) is homogeneous in the multi-limit if there exist a finite number of triplets $\left(r_{i}, \lambda_{i}, g_{i}\right.$ (respectively $\left.f_{i}\right)$ ) for which the function (respectively the vector field $f$, the system (1)) is $\left(r_{i}, \lambda_{i}, g_{i}\right.$ (respectively $\left.f_{i}\right)$ )-locally homogeneous for each index $i$. 
The following formulas give an example for choice of locally approximating functions for any $0<\lambda_{0}<+\infty$ and $x \in S_{r}[8]:$

$$
h_{0}(x)=\lambda_{0}^{-d_{0}} h\left(\Lambda_{r, 0} x\right), f_{0}(x)=\lambda_{0}^{-d_{0}} \Lambda_{r, 0}^{-1} f\left(\Lambda_{r, 0} x\right),
$$

where $\Lambda_{r, 0}=\operatorname{diag}\left\{\lambda_{0}^{r_{i}}\right\}_{i=1}^{n}$. Moreover, the approximating functions can be chosen homogeneous:

$$
\begin{gathered}
h_{0}(x)=\|x\|_{r}^{d} \lambda_{0}^{-d_{0}} h\left(\Lambda_{r, 0} \Lambda_{\|x\|}^{-1} x\right), \\
f_{0}(x)=\|x\|_{r}^{d} \lambda_{0}^{-d_{0}} \Lambda_{\|x\|} \Lambda_{r, 0}^{-1} f\left(\Lambda_{r, 0} \Lambda_{\|x\|}^{-1} x\right),
\end{gathered}
$$

where $\Lambda_{\|x\|}=\operatorname{diag}\left\{\|x\|_{r}^{r_{i}}\right\}_{i=1}^{n}$, provided that $h_{0}(0)$ and $f_{0}(0)$ are well defined. The proposed formulas do not cover two limit cases with $\lambda_{0}=0$ and $\lambda_{0}=+\infty$. For the case $\lambda_{0}=0$ at least one variant of the approximating dynamics can be pointed out for differentiable functions $h$ and $f$ with the property $h(0)=0, f(0)=0: h_{0}(x)=g^{\prime}(0) x, f_{0}(x)=$ $f^{\prime}(0) x$ for $r_{i}=1, i=\overline{1, n}$.

\section{Conditions of oscillation in the sense of Yakubovich}

The function $g: R^{n} \rightarrow R$ is called monotone if the condition $x_{1} \leq x_{1}^{\prime}, \ldots, x_{n} \leq x_{n}^{\prime}$ implies that everywhere either $g\left(x_{1}, \ldots, x_{n}\right) \leq g\left(x_{1}^{\prime}, \ldots, x_{n}^{\prime}\right)$ or $g\left(x_{1}, \ldots, x_{n}\right) \geq$ $g\left(x_{1}^{\prime}, \ldots, x_{n}^{\prime}\right)$.

Definition 7. [29], [7] The solution $x\left(t, x_{0}\right)$ with $x_{0} \in \mathbb{R}^{n}$ of the system (1) is called $\left[\pi^{-}, \pi^{+}\right]$-oscillation with respect to the output $\psi=\eta(x)$ (where $\eta: \mathbb{R}^{n} \rightarrow \mathbb{R}$ is a continuous monotone function) if the solution is defined for all $t \geq 0$ and

$$
\begin{gathered}
\underline{\lim }_{t \rightarrow+\infty} \psi(t)=\pi^{-} ; \varlimsup_{t \rightarrow+\infty} \psi(t)=\pi^{+} \\
-\infty<\pi^{-}<\pi^{+}<+\infty
\end{gathered}
$$

The solution $x\left(t, x_{0}\right)$ with $x_{0} \in \mathbb{R}^{n}$ of the system (1) is called oscillating, if there exist some output $\psi$ and constants $\pi^{-}, \pi^{+}$such that $x\left(t, x_{0}\right)$ is $\left[\pi^{-}, \pi^{+}\right]$-oscillation with respect to the output $\psi$. The forward complete system (1) is called oscillatory, if for almost all $x_{0} \in \mathbb{R}^{n}$ the solutions $x\left(t, x_{0}\right)$ of the system are oscillating. The oscillatory system (1) is called uniformly oscillatory, if for almost all $x_{0} \in \mathbb{R}^{n}$ for corresponding solutions $x\left(t, x_{0}\right)$ there exist output $\psi$ and constants $\pi^{-}, \pi^{+}$not depending on initial conditions.

In other words the solution $x\left(t, x_{0}\right)$ is oscillating if the output $\psi(t)=\eta\left(x\left(t, x_{0}\right)\right)$ is asymptotically bounded and there is no single limit value of $\psi(t)$ for $t \rightarrow+\infty$. The term "almost all solutions" is used to emphasize that generally the system (1) has a nonempty set of equilibrium points, thus there exists a set of initial conditions with zero measure such that the corresponding solutions are not oscillating. The notion of oscillations in the sense of Yakubovich is rather generic including periodical oscillations (limit cycles), quasi-periodical, recurrent and chaotic trajectories. The oscillating trajectories could be repelling being oscillating. The trajectories also could be unbounded, it is required to find a function of the state vector, that is bounded and admits certain requirements introduced in Definition 7 .

Theorem 8. [7] Let the system (1) have two locally Lipschitz continuous Lyapunov functions $V_{1}$ and $V_{2}$ fulfilling the following inequalities for all $x \in \mathbb{R}^{n}$ :

$v_{1}(\|x\|) \leq V_{1}(x) \leq v_{2}(\|x\|), v_{3}(\|x\|) \leq V_{2}(x) \leq v_{4}(\|x\|)$,

$v_{1}, v_{2}, v_{3}, v_{4} \in K_{\infty}$ and for some $0<X_{1}<v_{1}^{-1} \circ v_{2} \circ$ $v_{3}^{-1} \circ v_{4}\left(X_{2}\right)<+\infty$ :

$D V_{1}(x) f(x)>0$ for all $0<\|x\|<X_{1}$ and $x \notin \Xi$;

$D V_{2}(x) f(x)<0$ for all $\|x\|>X_{2}$ and $x \notin \Xi$,

where $\Xi \subset \mathbb{R}^{n}$ is a set with zero Lebesgue measure containing all equilibriums of the system, and

$\Omega \cap \Xi=\emptyset, \Omega=\left\{x: v_{2}^{-1} \circ v_{1}\left(X_{1}\right) \leq\|x\| \leq v_{3}^{-1} \circ v_{4}\left(X_{2}\right)\right\}$.

Then the system (1) is oscillatory.

Theorem 9. [8] Let the system (1) be $\left(r_{j}, \lambda_{j}, f_{j}\right)-$ homogeneous for $j=1,2$, the functions $f: \mathbb{R}^{n} \rightarrow \mathbb{R}^{n}$ and $f_{j}: \mathbb{R}^{n} \rightarrow \mathbb{R}^{n}, j=1,2$ be continuous and the locally approximating dynamical systems $\dot{x}=f_{j}(x), j=1,2$ have $r_{j}$-homogeneous and continuously differentiable Lyapunov functions $V_{j}: \mathbb{R}^{n} \rightarrow \mathbb{R}_{+}, \alpha_{1, j}(\|x\|) \leq V_{j}(x) \leq \alpha_{2, j}(\|x\|)$, $\alpha_{1, j}, \alpha_{2, j} \in \mathcal{K}_{\infty}$ for all $x \in \mathbb{R}^{n}$ and $j=1,2$. Let $\Xi \subset R^{n}$ be the set containing all equilibriums of the system (1). Let one of the following conditions hold:

- (i) $a_{1}=\inf _{y \in S_{r_{1}}} D V_{1}(y) f_{1}(y)>0, a_{2}=$ $-\sup _{y \in S_{r_{2}}} D V_{2}(y) f_{2}(y)>0$ and the sets

$$
\begin{gathered}
X_{j}=\left\{x \in \mathbb{R}^{n}: \alpha_{1, j}^{-1} \circ \alpha_{2, j}\left(m_{2} \underline{\lambda}_{j}\right)<\|x\|<\right. \\
\left.\alpha_{1, j}^{-1} \circ \alpha_{2, j}\left(m_{1} \bar{\lambda}_{j}\right)\right\}, j=1,2
\end{gathered}
$$

are connected and non empty where

$$
\sup _{\mathbf{y} \in S_{\mathbf{r}_{j}}}\left|D V_{j}(y)\left[\lambda^{-d} \Lambda_{r_{j}}^{-1} f\left(\Lambda_{r_{j}} y\right)-f_{j}(y)\right]\right|<a_{j}, j=1,2
$$

for all $\lambda \in\left(\underline{\lambda}_{j}, \bar{\lambda}_{j}\right), \underline{\lambda}_{j} \leq \lambda_{j} \leq \bar{\lambda}_{j}$ (such constants $\underline{\lambda}_{j}$, $\bar{\lambda}_{j}$ exist due to homogeneity assumption), the constants $m_{1}, m_{2}$ are defined in Lemma 2, and

$$
\begin{gathered}
\Omega \cap \Xi=\emptyset, \Omega=\Omega_{1} \cap \Omega_{2}, \\
\Omega_{1}=\mathbb{R}^{n} \backslash\left\{x \in \mathbb{R}^{n}:\|x\| \leq \alpha_{1,1}^{-1} \circ \alpha_{2,1}\left(m_{1} \bar{\lambda}_{1}\right)\right\}, \\
\Omega_{2}=\left\{x \in \mathbb{R}^{n}:\|x\| \leq \alpha_{1,2}^{-1} \circ \alpha_{2,2}\left(m_{2} \underline{\lambda}_{2}\right)\right\} ;
\end{gathered}
$$

- (ii) $a_{1}=-\sup _{y \in S_{r_{1}}} D V_{1}(y) f_{1}(y)>0, a_{2}=$ $\inf _{y \in S_{r_{2}}} D V_{2}(y) f_{2}(y)>0$ and the sets $X_{j}, j=1,2$ are connected and non empty where $\underline{\lambda}_{j} \leq \lambda_{j} \leq \bar{\lambda}_{j}$ are defined as for the case (i), and

$$
\begin{gathered}
\Omega \cap \Xi=\emptyset, \Omega=\left\{x \in \mathbb{R}^{n}: \alpha_{1,1}^{-1} \circ \alpha_{2,1}\left(m_{1} \bar{\lambda}_{1}\right) \leq\right. \\
\left.\|x\| \leq \alpha_{1,2}^{-1} \circ \alpha_{2,2}\left(m_{2} \underline{\lambda}_{2}\right)\right\} .
\end{gathered}
$$

Then the system (1) has oscillating trajectories into the set $\Omega$.

After introduction of these preliminary notions let us proceed with the main result presentation. 


\section{CONTROL DESIGN}

Consider an affine in control nonlinear system

$$
\dot{x}=f(x)+G(x) u, x \in \mathbb{R}^{n}, u \in \mathbb{R}^{m},
$$

where $f$ and the columns of $G$ are locally Lipschitz continuous vector fields, $f(0)=0$. There exist a lot of approaches devoting to the stabilizing control $u$ construction [16], [22] for (3), and some methods of anti-control design [3], [4], [27] (the controls granting the closed loop system with instability property).

Among approaches for stabilizing control design it is worth to mention CLF method [2], [26], [6], [5] that gives a universal formula for the control laws. For the homogeneous system (3) this approach has been developed in [9], [17], [18], [19].

Definition 10. [26] A continuously differentiable and positive definite function $V: \mathbb{R}^{n} \rightarrow \mathbb{R}_{+}$is called a CLF for the system (3) if for all $x \in \mathbb{R}^{n} \backslash\{0\}$,

$$
\inf _{u \in \mathbb{R}^{m}}\left\{a(x)+B(x)^{T} u\right\}<0,
$$

where $a(x)=D V(x) f(x), B(x)=[D V(x) G(x)]^{T}$. Such a CLF satisfies the Small Control Property (SCP) if for each $\epsilon>0$ there is a $\delta>0$ such that, if $x \neq 0$ satisfies $\|x\|<\delta$, then there is some $\|u\|<\epsilon$ such that

$$
a(x)+B(x)^{T} u<0 .
$$

It is possible to show [26] that a continuously differentiable and positive definite function $V: \mathbb{R}^{n} \rightarrow \mathbb{R}_{+}$is a CLF for the system (3) if for all $x \in \mathbb{R}^{n} \backslash\{0\}$ the property

$$
a(x)<0 \quad \text { if }\|B(x)\|=0
$$

holds. The SCP property is equivalent to the following one:

$$
\lim _{\|x\| \rightarrow 0} \frac{a(x)}{\|B(x)\|} \leq 0 .
$$

Now we are in position to introduce the new anti-control (destabilizing) Lyapunov function.

Definition 11. A continuously differentiable and positive definite function $V: \mathbb{R}^{n} \rightarrow \mathbb{R}_{+}$is called an Anti-control Lyapunov Function (ALF) for the system (3) if for all $x \in \mathbb{R}^{n} \backslash\{0\}$,

$$
\sup _{u \in \mathbb{R}^{m}}\left\{a(x)+B(x)^{T} u\right\}>0 .
$$

Such an ALF satisfies the Small Control Property (SCP) if for each $\epsilon>0$ there is a $\delta>0$ such that, if $x \neq 0$ satisfies $\|x\|<\delta$, then there is some $\|u\|<\epsilon$ such that

$$
a(x)+B(x)^{T} u>0 .
$$

Similarly, it is possible to conclude that a continuously differentiable and positive definite function $V: \mathbb{R}^{n} \rightarrow \mathbb{R}_{+}$is an ALF, if for all $x \in \mathbb{R}^{n} \backslash\{0\}$ the property

$$
a(x)>0 \quad \text { if } \quad\|B(x)\|=0
$$

holds. The SCP property for ALF is equivalent to the limit one:

$$
\lim _{\|x\| \rightarrow 0} \frac{a(x)}{\|B(x)\|} \geq 0
$$

Lemma 12. [17] If for the system (3) there exists a CLF $V: \mathbb{R}^{n} \rightarrow \mathbb{R}_{+}$, then the control

$$
\begin{gathered}
u(x)=-\phi_{1}[a(x), \| B(x)||] B(x), \\
\phi_{1}(a, b)= \begin{cases}\frac{a+\sqrt[p]{|a|^{p}+b^{2 q}}}{b^{2}} & \text { if } b \neq 0 ; \\
0 & \text { if } b=0\end{cases}
\end{gathered}
$$

for any $2 q \geq p>1, q>1$ is continuous for all $x \in \mathbb{R}^{n} \backslash\{0\}$ and ensures the system stabilization. If the function a and all elements of the vector function $B$ are r-homogeneous with degrees $d_{a}$ and $d_{B}$ respectively:

$$
a\left(\Lambda_{r} x\right)=\lambda^{d_{a}} a(x) ; B_{i}\left(\Lambda_{r} x\right)=\lambda^{d_{B}} B_{i}(x), i=\overline{1, m},
$$

then the control (4) is elementwise r-homogeneous with degree $d_{a}-d_{B}$ provided that it is possible to choose $2 d_{B} q=d_{a} p$. If the CLF $V$ is $r$-homogeneous with degree $d_{V}$ and the vector field $f$ and all columns of the matrix function $G$ are $r$-homogeneous with degrees $d_{f}$ and $d_{G}$ respectively:

$$
f\left(\Lambda_{r} x\right)=\lambda^{d_{f}} \Lambda_{r} f(x) ; G^{i}\left(\Lambda_{r} x\right)=\lambda^{d_{G}} \Lambda_{r} G^{i}(x), i=\overline{1, m},
$$

then the control (4) is elementwise r-homogeneous with degree $d_{f}-d_{G}$ and the closed loop system (3), (4) is $r-$ homogeneous with degree $d_{f}$ provided that $2\left(d_{G}+d_{V}\right) q=$ $\left(d_{f}+d_{V}\right) p$.

If furthermore $V$ satisfies the SCP, then the feedback control (4) is also continuous at the origin.

The last part of this lemma has been proven in [17] for the case $m=1$ only.

Lemma 13. If for the system (3) there exists an ALF $V$ : $\mathbb{R}^{n} \rightarrow \mathbb{R}_{+}$, then the control

$$
\begin{gathered}
u(x)=-\phi_{2}[a(x), \| B(x)||] B(x), \\
\phi_{2}(a, b)= \begin{cases}\frac{a-\sqrt[p]{|a|^{p}+b^{2 q}}}{b^{2}} & \text { if } b \neq 0 ; \\
0 & \text { if } b=0\end{cases}
\end{gathered}
$$

for any $2 q \geq p>1, q>1$ is continuous for all $x \in \mathbb{R}^{n} \backslash\{0\}$ and ensures the system instability. If the function $a$ and all elements of the vector function $B$ are r-homogeneous with degrees $d_{a}$ and $d_{B}$ respectively:

$$
a\left(\Lambda_{r} x\right)=\lambda^{d_{a}} a(x) ; B_{i}\left(\Lambda_{r} x\right)=\lambda^{d_{B}} B_{i}(x), i=\overline{1, m},
$$

then the control (5) is elementwise r-homogeneous with degree $d_{a}-d_{B}$ provided that it is possible to choose $2 d_{B} q=d_{a} p$. If the $A L F V$ is $r$-homogeneous with degree $d_{V}$ and the vector field $f$ and all columns of the matrix function $G$ are $r$-homogeneous with degrees $d_{f}$ and $d_{G}$ respectively:

$f\left(\Lambda_{r} x\right)=\lambda^{d_{f}} \Lambda_{r} f(x) ; G^{i}\left(\Lambda_{r} x\right)=\lambda^{d_{G}} \Lambda_{r} G^{i}(x), i=\overline{1, m}$, 
then the control (5) is elementwise r-homogeneous with degree $d_{f}-d_{G}$ and the closed loop system (3), (5) is $r$ homogeneous with degree $d_{f}$ provided that $2\left(d_{G}+d_{V}\right) q=$ $\left(d_{f}+d_{V}\right) p$.

If furthermore $V$ satisfies the SCP, then the feedback control (5) is also continuous at the origin.

Proof: The proof is excluded due to space limitations.

Together with the local homogeneity concept, these two lemmas may be used to propose a universal control formula for the system (3) stabilization/destabilization at a specified sphere around the origin.

Theorem 14. Assume that for the system (3):

(i) there exists a r-homogeneous CLF (ALF) $V_{0}: \mathbb{R}^{n} \rightarrow$ $\mathbb{R}_{+}, d_{V}=\operatorname{deg}_{r}\left(V_{0}\right)$; $d_{f}$;

(ii) the function $f$ is $\left(r, \lambda_{0}, f_{0}\right)$-homogeneous with degree

(iii) the columns $G^{i}, i=\overline{1, m}$ of the matrix function $G$ are $\left(r, \lambda_{0}, G_{0}^{i}\right)$-homogeneous with degree $d_{G}$;

(iv) there exist $2 q \geq p>1, q>1$ such that $2\left(d_{G}+d_{V}\right) q=$ $\left(d_{f}+d_{V}\right) p$.

Then the system (3) with the control (4) (the control (5)) for $a(x)=D V_{0}(x) f_{0}(x), B(x)=\left[D V_{0}(x) G_{0}(x)\right]^{T}$ is $\left(r, \lambda_{0}, F_{0}\right)$-homogeneous with degree $d_{f}$, where the vector field $F_{0}(x)=f_{0}(x)+G_{0}(x) u(x)$ is stable (unstable).

Proof: For the system $\dot{x}=f_{0}(x)+G_{0}(x) u$ all conditions of the lemma 12 (the lemma 13) are satisfied, the system $\dot{x}=$ $F_{0}(x)$ is stable (unstable) and $r$-homogeneous with degree $d_{f}$. Let us show, that the system (3) with the control (4) (the control (5)) is $\left(r, \lambda_{0}, F_{0}\right)$-homogeneous with degree $d_{f}$. For $F(x)=f(x)+G(x) u(x)$ by definition we have

$$
\begin{gathered}
\lambda^{-d_{f}} \Lambda_{r}^{-1} F\left(\Lambda_{r} x\right)=\lambda^{-d_{f}} \Lambda_{r}^{-1}\left[f\left(\Lambda_{r} x\right)+G\left(\Lambda_{r} x\right) u\left(\Lambda_{r} x\right)\right]= \\
\left.=\lambda^{-d_{f}} \Lambda_{r}^{-1} f\left(\Lambda_{r} x\right)+\lambda^{-d_{G}} \Lambda_{r}^{-1} G\left(\Lambda_{r} x\right) \lambda^{d_{G}-d_{f}} u\left(\Lambda_{r} x\right)\right] .
\end{gathered}
$$

Since

$$
\begin{gathered}
u\left(\Lambda_{r} x\right)=\lambda^{d_{f}-d_{G}} u(x), \lim _{\lambda \rightarrow \lambda_{0}}\left(\lambda^{-d_{f}} \Lambda_{r}^{-1} f\left(\Lambda_{r} x\right)-f_{0}(x)\right)=0, \\
\lim _{\lambda \rightarrow \lambda_{0}}\left(\lambda^{-d_{G}} \Lambda_{r}^{-1} G\left(\Lambda_{r} x\right)-G_{0}(x)\right)=0
\end{gathered}
$$

we obtain $\lim _{\lambda \rightarrow \lambda_{0}}\left(\lambda^{-d_{f}} \Lambda_{r}^{-1} F\left(\Lambda_{r} x\right)-F_{0}(x)\right)=0$.

For $0<\lambda_{0}<+\infty$, a variant of homogeneous approximating functions $f_{0}$ and $G_{0}^{i}, i=\overline{1, m}$ is given in (2). Any other approach (backstepping, forwarding or feedback linearization) generating a $r$-homogeneous control may substitute the CLF/ALF controls (4), (5) in this theorem under conditions (i)-(iii). Owing the framework of homogeneity in the multilimit, Theorem 14 provides an approach to design oscillating systems.

Corollary 15. Let for some $r_{k}, k=\overline{0, N}, 0<N<+\infty$ and $0 \leq \lambda_{0}<\ldots<\lambda_{N} \leq+\infty$ for the system (3) the following properties be true:

(i) there exist $r_{2 j}$-homogeneous CLFs (ALFs) $V_{2 j}: \mathbb{R}^{n} \rightarrow$ $\mathbb{R}_{+}$and $r_{2 j+1}$-homogeneous ALFs (CLFs) $V_{2 j+1}: \mathbb{R}^{n} \rightarrow$
$\mathbb{R}_{+}$for $j=\overline{0, N / 2}$, define $h_{2 j}=1, h_{2 j+1}=2\left(h_{2 j}=2\right.$, $\left.h_{2 j+1}=1\right)$ and $d_{V, k}=\operatorname{deg}_{r_{k}}\left(V_{k}\right)$ for $k=\overline{0, N}$;

(ii) the function $f$ is $\left(r_{k}, \lambda_{k}, f_{k}\right)$--homogeneous with degree $d_{f, k}, k=\overline{0, N}$;

(iii) the columns $G^{i}, i=\overline{1, m}$ of the matrix function $G$ are $\left(r_{k}, \lambda_{k}, G_{k}^{i}\right)$-homogeneous with degree $d_{G, k}, k=\overline{0, N}$;

(iv) for all $k=\overline{0, N}$ there exist $2 q_{k} \geq p_{k}>1, q_{k}>1$ such that $2\left(d_{G, k}+d_{V, k}\right) q_{k}=\left(d_{f, k}+d_{V, k}\right) p_{k}$;

$(v)$ the vector fields $f$ and $G^{i}, i=\overline{1, m}$ are linearly independent for all $x \in \mathbb{R}^{n} \backslash\{0\}$.

Then the system (3) with the control

$$
u(x)=-\sum_{k=0}^{N}\left[\varrho_{k}\left(\|x\|_{k}, \lambda_{k}\right) \phi_{h_{k}}\left[a_{k}(x),\left\|B_{k}(x)\right\|\right] B_{k}(x)\right],
$$

$$
a_{k}(x)=D V_{k}(x) f_{k}(x), B_{k}(x)=\left[D V_{k}(x) G_{k}(x)\right]^{T},
$$

has different oscillating trajectories into the sets $X_{k}=$ $\left\{x \in \mathbb{R}^{n}: \lambda_{k}<\|x\|<\lambda_{k+1}\right\}, k=\overline{0, N-1}$, where the continuous weighting functions $\varrho_{k}\left(\lambda_{k}, \lambda_{k}\right)=1$ and $\sum_{k=0}^{N} \varrho_{k}\left(\|x\|_{k}, \lambda_{k}\right)=1$ for any $x \in \mathbb{R}^{n}$.

Proof: If the vector fields $f$ and $G^{i}, i=\overline{1, m}$ are linearly independent for all $x \in \mathbb{R}^{n} \backslash\{0\}$, then for any control the system has the single equilibrium at the origin. For each $k=\overline{0, N}$ the conditions of Theorem 14 hold and the system (3) with the control $u_{k}(x)=\phi_{h_{k}}\left[a_{k}(x),\left\|B_{k}(x)\right\|\right] B_{k}(x)$ is stable or unstable and locally homogeneous. The function $\varrho_{k}\left(\|x\|_{k}, \lambda_{k}\right)$ equals 1 for $\|x\|_{r_{k}}=\lambda_{k}$ and it uniformly decays to zero on leaving this set (due to the normalization condition $\left.\sum_{k=0}^{N} \varrho_{k}\left(\|x\|_{k}, \lambda_{k}\right)=1\right)$. Therefore, the closed-loop system (3), (6) is $\left(r_{k}, \lambda_{k}, F_{k}\right)$-homogeneous for $F_{k}(x)=f_{k}(x)+G_{k}(x) u_{k}(x)$. The oscillating properties follow by application of Theorem 9 (all conditions of this theorem hold for each paire $2 j, 2 j+1, j=\overline{0, N / 2}$ ).

Note that there exist constants $0<\chi_{k}<+\infty, k=\overline{0, N}$ such that the choice

$$
\varrho_{k}\left(\|x\|_{k}, \lambda_{k}\right)=e^{-\chi_{k} \nu_{k}\left(\|x\|_{r_{k}}, \lambda_{k}\right)^{2}}, k=\overline{0, N}
$$

is admissible, where $\nu_{k}(s, \lambda)=s-\lambda, k=\overline{0, N}$ and if $\lambda_{N}=+\infty$ then

$$
\nu_{N}(s,+\infty)= \begin{cases}0 & \text { if } s-\lambda_{N}^{\prime} \geq 0 \\ s-\lambda_{N}^{\prime} & \text { if } s-\lambda_{N}^{\prime}<0\end{cases}
$$

for some $\lambda_{N-1}<\lambda_{N}^{\prime}<+\infty$. The term $e^{-\chi_{k} \nu_{k}\left(\|x\|_{r_{k}}, \lambda_{k}\right)^{2}}$ can be replaced with any other type of weighting functions (polynomial, for instance). For this choice of the functions $\varrho_{k}, k=\overline{0, N}$, the normalization condition holds with some error (dependent on $\chi_{k}$ ), however the arguments of Theorem 9 remain valid.

Example 16. Consider a bilinear planar system:

$$
\begin{gathered}
\dot{x}=f(x)+g(x) u, x \in \mathbb{R}^{2}, u \in \mathbb{R}, \\
f(x)=\left[x_{2}-2 x_{1}\right]^{T}, g(x)=\left[\begin{array}{ll}
x_{1} & x_{2}
\end{array}\right]^{T} .
\end{gathered}
$$


The vector fields $f$ and $g$ are linearly independent for all $x \in \mathbb{R}^{2} \backslash\{0\}$. Choose $\lambda_{0}=0$ and $r_{0}=\left[\begin{array}{ll}1 & 1\end{array}\right]$, then due to linearity of $f$ and $g$ we obtain $f_{0}(x)=f(x), g_{0}(x)=g(x)$. Take $V_{0}(x)=\left(x_{1}+x_{2}\right)^{2}+x_{2}^{2}$, then $a_{0}(x)=2\left(x_{1}+x_{2}\right)\left(x_{2}-\right.$ $\left.4 x_{1}\right)-4 x_{1} x_{2}, b_{0}(x)=2\left(x_{1}+x_{2}\right)^{2}+2 x_{1} x_{2}$ and $V_{0}$ is a CLF. Since $d_{f, 0}=0, d_{g, 0}=0$ and $d_{V, 0}=2$, pick $q_{0}=1$ and $p_{0}=2$. Next, choose $\lambda_{1}=2$ and $r_{1}=\left[\begin{array}{ll}1 & 2\end{array}\right]$. According to (2) we obtain

$$
f_{1}(x)=\left[x_{2}-0.5\|x\|_{r_{1}}^{2} x_{1}\right]^{T}, g_{1}(x)=g(x)
$$

for $d_{f, 1}=1$ and $d_{g, 1}=0$. For $d_{V, 1}=4$ take $V_{1}(x)=$ $x_{2}^{2}+\left(x_{1}\left|x_{1}\right|+x_{2}\right)^{2}$, then

$$
\begin{gathered}
a_{1}(x)=2\left(x_{1}\left|x_{1}\right|+x_{2}\right)\left(2\left|x_{1}\right| x_{2}-0.5\|x\|_{r_{1}}^{2} x_{1}\right)-\|x\|_{r_{1}}^{2} x_{1} x_{2}, \\
b_{1}(x)=2\left(x_{1}\left|x_{1}\right|+x_{2}\right)\left(2\left|x_{1}\right| x_{1}+x_{2}\right)+2 x_{2}^{2}
\end{gathered}
$$

and $V_{1}$ is an ALF. Therefore, $q_{1}=1.25$ and $p_{1}=2$ is an admissible choice. Finally, let $\lambda_{2}=+\infty$ and $r_{2}=$ [11], then again $f_{2}(x)=f(x), g_{2}(x)=g(x), V_{2}(x)=\left(x_{1}+x_{2}\right)^{2}+x_{2}^{2}$ is a CLF, $a_{2}(x)=a_{0}(x), b_{2}(x)=b_{0}(x), q_{2}=1$ and $p_{2}=2$. In the control (6) we put (7) with $\chi_{k}=5, k=0,1,2$. The results of the system simulation are shown in Fig. 1, the system has two limit cycles (the inner is unstable and the outer one is stable).

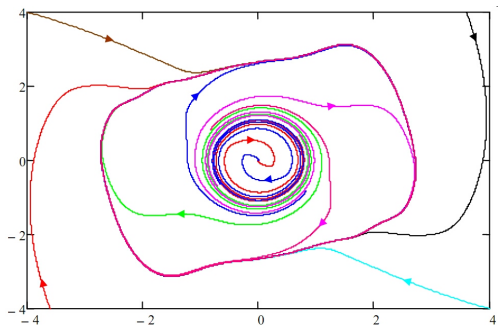

Fig. 1. Trajectories of the closed-loop system.

\section{CONCLUSION}

The paper extends the CLF approach for anti-control analysis and design, for that the ALF concept is introduced. The universal formula for destabilizing control algorithms is proposed. Applying the local homogeneity concept, the control law for stabilization/destabilization of nonlinear systems around a sphere is presented. Finally, the universal formulas for stabilizing/destabilizing controls are used for oscillating system design with multiple limit cycles. The results are illustrated by example of computer simulation.

\section{REFERENCES}

[1] V. Andrieu, L. Praly, and A. Astolfi. Homogeneous approximation, recursive observer design, and output feedback. SIAM J. Control Optimization, 47(4):1814-1850, 2008.

[2] Z. Artstein. Stabilization with relaxed control. Nonlinear Analysis, 7:1163-1173, 1983.

[3] G. Chen, editor. Controlling Chaos and Bifurcations in Engineering Systems. CRC, Boca Raton, FL, 1999.

[4] G. Chen and Y. Shi. Introduction to anti-control of discrete chaos: theory and applications. Philos Transact A Math Phys Eng Sci., 364(9):2433-2447, 2006.
[5] D. Efimov. A condition of clf existence for affine systems. In Proc. of 41th IEEE Conference on Decision and Control, pages 1882-1887, Las Vegas, 12 2002. IEEE.

[6] D. Efimov. Universal formula for output asymptotic stabilization. In Proc. of 15th IFAC World Congress, Barselona, Spain, 7 2002. IFAC.

[7] D. Efimov and A.L. Fradkov. Oscillatority of nonlinear systems with static feedback. SIAM J. Control Optimization, 48(2):618-640, 2009.

[8] D. Efimov and W. Perruquetti. Oscillations conditions in homogenous systems. In Proc. IFAC NOLCOS Symp., pages 1379-1384, Bologna, 2010.

[9] L. Faubourg and J.-B. Pomet. Control lyapunov functions for homoge-

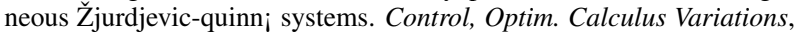
5:293-311, 2000.

[10] M. Fliess, J. Lévine, P. Martin, and P. Rouchon. On differentially flat non linear systems. In Proceedings of IFAC NOLCOS Conf., Bordeaux, France, 1992.

[11] M. Fliess, J. L. Lévine, P. Martin, and P. Rouchon. Flatness and defect of non-linear systems: introductory theory and examples. International Journal of Control, 61(6):1327-1361, 1995.

[12] A.L. Fradkov, I.V. Miroshnik, and V.O. Nikiforov. Nonlinear and adaptive control of complex systems. Kluwer, 1999.

[13] H. Hermes. Nilpotent and high-order approximations of vector field systems. SIAM Review, 33(2):238-264, 1991.

[14] A. Isidori. Nonlinear control systems: An Introduction. SpringerVerlag, Berlin, 2nd edition, 1989.

[15] P.V. Kokotović and M. Arcak. Constructive nonlinear control: a historical perspective. Automatica, 37:637-662, 2001.

[16] M. Krstić, I. Kanellakopoulos, and P.V. Kokotović. Nonlinear and Adaptive Control Design. Wiley \& Sons, Inc., 1995.

[17] E. Moulay. Stabilization via homogeneous feedback controls. Automatica, 44(11):2981-2984, 2008.

[18] E. Moulay and W. Perruquetti. Finite time stability and stabilization of a class of continuous systems. J. Mathematical Analysis Applications, 323(2):1430-1443, 2006.

[19] N. Nakamura, H. Nakamura, Y. Yamashita, and H. Nishitani. Homogeneous stabilization for input affine homogeneous systems. IEEE Trans. Aut. Contr., 54(9):2271-2275, 2009.

[20] L. Rosier. Homogeneous lyapunov function for homogeneous continuous vector field. Systems \&Control Lett., 19:467-473, 1992.

[21] V.V. Rumyantsev and A.S. Oziraner. Stability and stabilization of motion with respect to part of variables. Nauka, Moscow, 1987.

[22] R. Sepulchre, M. Jankovic, and P.V. Kokotović. Constructive nonlinear control. Springer-Verlag, NY, 1997.

[23] A.S. Shiriaev and C. Canudas de Wit. Virtual constraints: a tool for orbital stabilization of nonlinear systems theory. In Proc. 6th IFAC Symposium NOLCOS 2004, pages 1355-1360, Stuttgart, 2004. IFAC.

[24] A.S. Shiriaev and A.L. Fradkov. Stabilization of invariant sets for nonlinear non-affine systems. Automatica, 36:1709-1715, 2000

[25] R. Skjetne, A.R. Teel, and P.V. Kokotović. Nonlinear maneuvering with gradient optimization. In Proc. of IEEE CDC 2001, pages 39263931. IEEE, 2001.

[26] E.D. Sontag. A "universal" construction of arstein's theorem on nonlinear stabilization. Systems \& Control Letters, 12:542-550, 1989.

[27] A. Vanecek and S. Celikovsky. Control Systems: From Linear Analysis to Synthesis of Chaos. Prentice-Hall, London, 1996.

[28] V.I. Vorotnikov. Partial Stability and Control. Birkhauser, Boston, 1998.

[29] V.A. Yakubovich. Frequency oscillations conditions in nonlinear systems with stationary single nonlinearity. Siberian Math. J., 14(2), 1973. 